\title{
PERFORMANCE OF POULTRY LITTER BASED COMPOST ON MORPHO-PHYSIOLOGICAL CHARACTERS AND YIELD OF T. AMAN RICE AND SOIL FERTILITY
}

\author{
A.K.M.M.B. Chowdhury ${ }^{1}$, T.S. Tudu ${ }^{1}$, M.A. Shohag ${ }^{1}$, M.A. Hossain ${ }^{1}$ and M.Z. Islam ${ }^{2}$ \\ ${ }^{1}$ Department of Crop Physiology and Ecology, Hajee Mohammad Danesh Science and \\ Technology University (HSTU), Dinajpur, Bangladesh. \\ ${ }^{2}$ Division of Plant Breeding, Bangladesh Wheat and Maize Research Institute(BWMRI),Dinajpur, Bangladesh. \\ Corresponding E-mail: minarbari07@gmail.com
}

(Received: 18 March, 2020, Accepted: 19 August, 2020)

Keywords: Rice varieties, fertilization, soil health, yield, yield contributing characters

\begin{abstract}
An experiment was carried out at the Research Field of Crop Physiology and Ecology Department, Hajee Mohammad Danesh Science and Technology University, Dinajpur from July to November 2018. The study evaluated the effects of poultry litter based composts on morpho-physiological, yield and yield contributing characters of transplanted aman rice (var.Binadhan-7, BRRI dhan56, BRRI dhan66) and soil fertility. The experiment was laid out in a two factorial complete randomized design $(\mathrm{CRD})$ with three replications. Factor $\mathrm{A}$ included three varieties (Binadhan-7: $\mathrm{V}_{1}$, BRRI dhan56: $\mathrm{V}_{2}$, and BRRI dhan66: $\mathrm{V}_{3}$ ) and factor $\mathrm{B}$ comprised of three fertilization levels viz. control (inorganic)- $F_{1}$, poultry litter based compost 20 ton ha ${ }^{-1}: F_{2}$, and 30 ton ha ${ }^{-1}: F_{3}$. The performances of plant growth parameters such as plant height, tiller number, leaf number, leaf area, SPAD value, as well as yield and yield contributing parameters, and soil chemical properties were measured. Varieties and fertilizer levels significantly influenced most of the morphophysiological traits and yield and yield contributing characters. The tallest plant was recorded from $\mathrm{V}_{2}$ variety (BRRI dhan56). The highest leaf number hill-1 ${ }^{-1} 80.89$ at 30 DAT) was recorded from $\mathrm{V}_{3}$ (BRRI dhan66). The highest number of tiller was produced by the interaction of compost $F_{2}\left(20 \mathrm{t} \mathrm{ha}^{-1}\right)$ with $\mathrm{V}_{1}$ (Binadhan-7). The highest effective tiller number (13.67) hill ${ }^{-1}$ wasrecorded from $F_{2}$ (compost 20 t ha ${ }^{-1}$ ) with $\mathrm{F}_{1}$ (Binadhan-7) at maturity. The longest panicle $(24.16 \mathrm{~cm})$ was found in $\mathrm{F}_{1} \mathrm{~V}_{1}$ (inorganic with Binadhan-7) treatment. The highest 1000-grain weight $(23.57 \mathrm{~g})$ was recorded from $\mathrm{F}_{3} \mathrm{~V}_{3}$ treatment (compost-30 $\mathrm{t}$ ha ${ }^{-1}$ with BRRI dhan66). The maximum harvest index was recorded from $\mathrm{F}_{1}$ (inorganic) with BRRI dhan66. Soil fertility status increased with higher dose poultry litter based compost $\left(30 \mathrm{t} \mathrm{ha}^{-1}\right)$.
\end{abstract}

\section{Introduction}

Rice (Oryza sativa L.) is the principal food crop in Bangladesh, and about $74.85 \%$ of the total arable lands are used for rice (aus, aman and boro) cultivation (BBS, 2018). Rice plays a dominant role in Bangladesh agriculture sinceit covers 74.85 percent of the total cropped area (BBS, 2018). The yield of rice is low (3.072 t ha-1) in Bangladesh compared to other ricegrowing countries (BBS, 2018). It has already been reported that decreasing organic matter in farm soils has caused significant yield reductions (Shelly et al., 2016). Almost 85\% of the population of Bangladesh lives in rural areas having the main occupation of farming. The 
government of Bangladesh has given the highest priority to increase food availability in the country (Saha et al., 2009). One of the methods to reach this goal regarding increasing the food sufficiency is the reduction of the yield gap of the food grain per unit area. A modern variety of transplant aman can be planted intensively to augment income and welfare for low-income farmers (Sarker et al., 2013). The depleted soil fertility is a major constraint to higher crop production in Bangladesh. Rice-rice is the most common cropping system in Bangladesh. Continuous practicing of this highly exhaustive cropping sequence in most irrigated fertile lands resulted in the decline of soil physio-chemical conditions in general, particularly soil organic matter (SOM) content (Liza et al., 2014 and Hossaen et al., 2011).

It is known that inorganic fertilizers supply only nutrients in the soil, but organic manure supplies nutrients as well as improves soil quality. The long term impact of chemical fertilizers on soils and the environment is harmful (Tilahun et al., 2013, Diacono and Montemurro, 2010 and Golabi et al., 2007). The use of unbalanced nutrients in the soils may be harmful in the long run causing soils an unproductive one. Indeed, sustainable production of crops cannot be maintained by using only chemical fertilizers, and similarly, it is not possible to obtain higher crop yield by using organic manure alone (Saha, 2014 and Bair, 1990). A judicious combination of organic and inorganic sources of nutrients is necessary for sustainable agriculture that can ensure food production with high quality (Islam et al., 2014 and Nambiar, 1991). Integrated use of organic manure and chemical fertilizers would be quite promising not only in providing high stability in production but also in maintaining better soil fertility (Chowdhury et al., 2020 and Alam, 2017). Poultry manure is another good source of nutrients in the soil. Meelu and Singh (1991) showed that $4 \mathrm{t} \mathrm{ha}^{-1}$ poultry manure along with $60 \mathrm{~kg} \mathrm{~N}^{-1}$ as urea produce grain yield of crop similar to that with $120 \mathrm{~kg} \mathrm{Nha}^{-1}$ as urea alone. But direct application of poultry manure has harmful effects on soil health (Urra et al., 2019). That's why it needs to be composted. Poultry litter based compost can supply a substantial amount of plant nutrients and, therefore, can contribute to crop yields (Chowdhury et al., 2013, Kobra, 2016; Najafi and Abbasi, 2013).

Therefore, it is necessary to use fertilizer and manure in an integrated way to obtain sustainable crop yield without declining soil fertility. Applications of both chemical and organic fertilizers need to be applied for the improvement of soil physical properties and supply of essential plant nutrients for higher yield. The present investigation was, therefore, undertaken to develop a suitable integrated dose of poultry litter based compost for rice (var.Binadhan-7, BRRI dhan56, BRRI dhan66) and to observe the effects of different levels of inorganic fertilizers and poultry litter based compost on the yield and yield components of rice (var. Binadhan-7, BRRI dhan56, BRRI dhan66) as well as soil fertility.

\section{Materials and Methods}

The experiment was conducted to find out the effect of fertilizer and manure on the yield of $T$. aman rice. It was conducted during the period from July to November 2018 in the wet season. The location of the site is Crop Physiology and Ecology Research Field, Hajee Mohammad Danesh Science and Technology University (HSTU), Dinajpur. The experiment was laid out in a two factorial complete randomized design (CRD) with three replications.

Factor-A: Three varieties were $\mathrm{V} 1$ = Binadhan-7, $\mathrm{V}_{2}=\mathrm{BRRI}$ dhan56, $\mathrm{V}_{3}=\mathrm{BRRI}$ dhan 66 and

Factor-B: Three fertilization treatment were: $\mathrm{F} 1=$ Control (inorganic), $\mathrm{F}_{2}=$ compost $20 \mathrm{t} \mathrm{ha}^{-1}$, $\mathrm{F}_{3}=$ compost $30 \mathrm{tha}^{-1}$.

The soil was collected from the arable land of Crop physiology and Ecology Research Field, Hajee Mohammad Danesh Science and Technology University (HSTU), Dinajpur from a depth 
of $15 \mathrm{~cm}$. The collected soil was sundried for a week, and the weeds were removed. Fifteenkilogram soil was taken into each pot. Thus the pots were ready for planting. The pots were placed in the selected space of the research field of Crop Physiology and Ecology, HSTU, Dinajpur in 18 rows and 3 columns. The row to row and column to column distance was maintained properly.

The amounts of N, P, K, S and Zn fertilizers required per pot (control) were calculated as per the weight of soil. The full amount of TSP, MoP, gypsum and zinc sulphate was applied as basal dose before transplanting of rice seedlings for $F_{1}$ fertilization (control). Poultry litter based composts were applied $150 \mathrm{~g} \mathrm{pot}^{-1}$ for $\mathrm{F}_{2}$ fertilization and $225 \mathrm{~g} \mathrm{pot}^{-1}$ for $\mathrm{F}_{3}$ fertilization. The compost used in this research was made by Kobra (2016). Composting material was poultry manure, sawdust, rice husk and ash. Chemical compositions of poultry litter based compost were $\mathrm{N}-1.6 \%, \mathrm{P}-1.21 \%$, and K-0.35\% (Kobra, 2016). The seedlings of rice (var. Binadhan-7, BRRI dhan56and BRRI dhan66) were collected from BADC, Dinajpur. During seedling growing, no fertilizers were used. Proper water and pest management practices were followed whenever required. Forty days old seedlings of rice were carefully transplanted on July 23, 2018in well puddle pots. Three seedlings were sown in each pot. Intercultural operations were done to ensure the normal growth of the crop. Plant protection measures were followed as and when necessary. Soil N, P, K, S, pH, organic matter and organic carbon were measured. Soil analysis was done following standard methods at SRDI, Nashipur, Dinajpur. The data obtained for different parameters were statistically analyzed to find out the significant difference between chemical fertilizers and poultry litter based composts on the yield of transplant aman rice. The mean values of all the characters were calculated and analysis of variance was performed by MSTAT-C statistical software. The significance of the differences among the treatment means was estimated by Duncan's Multiple Range Test (DMRT) at 5\% level of probability (Gomez and Gomez, 1984).

\section{Results and Discussion}

The experiment was conducted to find out the effect of fertilizer and manure on the yield of $\mathrm{T}$. aman rice in different soil. The results are presented and discussed in the Tables and possible interpretations are given under the following headings.

\section{Plant height}

The effects of poultry litter based composts on plant height at different days after transplanting (DAT) were statistically significant (Table 1). Results showed that plant height increased with various doses of compost. The tallest plant was recorded in BRRI dhan56 at 30 DAT where the lengths were $74.22,99.11,101.3,101.6$ and $103.6 \mathrm{~cm}$ at maximum vegetative, booting, anthesis, and maturity stages, respectively. The shortest plant was recorded from Binadhan-7 where the lengths of the plants were $63.78,78.78,85.78,88.78$ and $91.22 \mathrm{~cm}$ at 30 DAT, maximum vegetative, booting, anthesis and maturity stages respectively. The tallest plant was recorded from $\mathrm{F}_{1}$ fertilization (inorganic) $(72.78 \mathrm{~cm}$ at $30 \mathrm{DAT})$. The tallest plant was recorded from $\mathrm{F}_{3}$ fertilization (compost-30t ha $\left.{ }^{-1}\right)(92.22$ and $101.1 \mathrm{~cm}$ at vegetative and booting stages, respectively. And the tallest plant was recorded from $\mathrm{F}_{1}$ fertilization (inorganic) (95.44 and 98.11 $\mathrm{cm}$ at anthesis and maturity stages, respectively. The shortest plants were recorded from $\mathrm{F}_{2}$ fertilization (compost-20 $\left.\mathrm{t} \mathrm{ha}^{-1}\right)(64.67,85.11,88.00,94$ and $96 \mathrm{~cm}$ at 30 DAT, maximum vegetative, booting, anthesis and maturity stages, respectively). Plant height was significantly affected by the interaction between fertilization and different varieties (Table 1). Results indicated that the tallest plants $(84.67,104.7,108,105.3$ and $107.3 \mathrm{~cm}$ at 30 DAT, maximum 
vegetative, booting, anthesis and maturity stages, respectively were found in $\mathrm{F}_{2}$ (compost $20 \mathrm{t}$ $\mathrm{ha}^{-1}$ ) fertilization with variety $V_{1}$ (Binadhan-7), and the shortest plants $(61.33 \mathrm{~cm}$ at 30 DAT) from $\mathrm{F}_{3} \mathrm{~V}_{2}$. Inorganic fertilizer may help in initial plant height enhancement due to quick nutrient availability, but later on, similar results were found by the application of other treatments (poultry litter based composts). A similar result was also reported by Hosaain et al. (2010) and Buri et al. (2006).

\section{Number of leaves hill-1}

The effects of poultry litter based composts on leaf number hill-1 at different days after transplanting (DAT) were statistically insignificant (Table 2). Results showed that leaf number hill1 increased with minimum different doses of compost. The highest leaf number hill ${ }^{-1}(80.89$ at 30 DAT) was recorded from $V_{3}$. The highest leaf number hill ${ }^{-1}$ at vegetative and booting were recorded from $V_{2}$ (BRRI dhan56) and the highest leaf number hill ${ }^{-1}$ at anthesis was recorded from $V_{1}$ (Binadhan-7). The lowest leaf number hill ${ }^{-1}$ was recorded from $V_{3}$ (54.56) at anthesis. The different fertilization doses influenced the leaf number hill ${ }^{-1}$. The highest number of leaves hill ${ }^{-1}$ was found from $F_{3}$ and the lowest one was for $F_{2}$ at anthesis. Leaf number hill-1 was insignificant.

\section{Number of tiller hill ${ }^{-1}$}

The effects of poultry litter based composts on tiller hill ${ }^{-1}$ at different days after transplanting (DAT) were statistically significant (Table 3). Results showed that the number of tillers hill-1 increased with various doses of compost. The highest number of tiller number hill ${ }^{-1}$ was recorded from $\mathrm{V}_{2}$ variety (BRRI dhan56) $(15.33,14.89,15.56,13.11$ and 14.11 at 30 DAT, maximum vegetative, booting, anthesis and maturity stages, respectively). The shortest plant was recorded from $\mathrm{V}_{1}$ variety (Binadhan-7) $(13,13.44,14.89,12.78$ and 13.44 at 30 DAT, maximum vegetative, booting, anthesis and maturity stages, respectively). The highest tillers number hill ${ }^{-1}$ were recorded from $F_{1}$ (inorganic) (15.33, 15.33, 13.67 and 15.44 at 30DAT, maximum vegetative, anthesis and maturity stages respectively). The highest tillers number hill ${ }^{-1}$ were recorded from $\mathrm{F}_{3}$ (compost-30 $\mathrm{t} \mathrm{ha}^{-1}$ ) (16.22 at booting stage). The lowest tillers number hill-

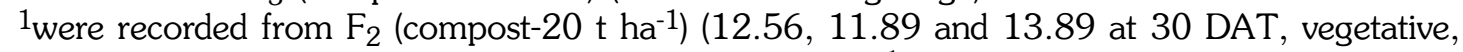
booting stages, respectively). The lowest tiller number hill ${ }^{-1}$ was recorded from $\mathrm{F}_{3}$ (compost-30 t $\left.\mathrm{ha}^{-1}\right)$ (11.78 and 12.33 at anthesis and maturity stages, respectably).

Table 1. Effect of fertilization on plant height of different rice varieties at different stages

\begin{tabular}{|c|c|c|c|c|c|}
\hline \multirow{2}{*}{$\begin{array}{l}\text { Treatments } \\
\text { Varieties }\end{array}$} & \multicolumn{5}{|c|}{$\begin{array}{l}\text { Plant height }(\mathrm{cm}) \\
\end{array}$} \\
\hline & $30 \mathrm{DAT}$ & Vegetative & Booting & Anthesis & Maturity \\
\hline $\mathrm{V}_{1=}$ Binadhan -7 & $63.78 b$ & $78.78 b$ & $85.78 b$ & $88.78 b$ & $91.22 b$ \\
\hline $\mathrm{V}_{2=} \mathrm{BRRI}$ dhan 56 & $74.22 \mathrm{a}$ & $99.11 \mathrm{a}$ & $101.3 a$ & $101.6 a$ & $103.6 a$ \\
\hline $\mathrm{V}_{3=} \mathrm{BRRI}$ dhan66 & $65.11 b$ & $90.67 a$ & $98.56 a$ & $94.22 \mathrm{ab}$ & $97 a b$ \\
\hline Level of significance & ** & *** & $*$ & $* *$ & $* *$ \\
\hline \multicolumn{6}{|c|}{ Fertilization } \\
\hline $\mathrm{F}_{1}=$ Inorganic & $72.78 a$ & 91.22 & $96.56 \mathrm{ab}$ & 95.44 & 98.11 \\
\hline $\mathrm{F}_{2}=$ Compost 20 tha $\mathrm{a}^{-1}$ & $64.67 b$ & 85.11 & $88.00 \mathrm{~b}$ & 94.00 & 96.00 \\
\hline $\mathrm{F}_{3}=$ Compost 30 tha $\mathrm{a}^{-1}$ & $65.67 b$ & 92.22 & 101.10a & 95.11 & 97.67 \\
\hline Level of significance & $* *$ & NS & $*$ & NS & NS \\
\hline \multicolumn{6}{|c|}{ Interaction } \\
\hline $\mathrm{V}_{1}$ & $64.00 \mathrm{bc}$ & $82.33 b c$ & $90.67 \mathrm{ab}$ & $92.33 \mathrm{~cd}$ & $96.33 b c$ \\
\hline
\end{tabular}




\begin{tabular}{ccccccc}
\hline Treatments & \multicolumn{5}{c}{ Plant height (cm) } \\
\hline $\mathrm{F}_{1}$ & $\mathrm{~V}_{2}$ & $62.67 \mathrm{bc}$ & $72.00 \mathrm{c}$ & $71.00 \mathrm{~b}$ & $86.33 \mathrm{~d}$ & $88.00 \mathrm{~d}$ \\
& $\mathrm{~V}_{3}$ & $64.67 \mathrm{bc}$ & $82.00 \mathrm{bc}$ & $95.67 \mathrm{a}$ & $87.67 \mathrm{~d}$ & $89.33 \mathrm{~cd}$ \\
& $\mathrm{~V}_{1}$ & $84.67 \mathrm{a}$ & $104.7 \mathrm{a}$ & $108.00 \mathrm{a}$ & $105.3 \mathrm{a}$ & $107.30 \mathrm{a}$ \\
$\mathrm{F}_{2}$ & $\mathrm{~V}_{2}$ & $70.00 \mathrm{~b}$ & $90.67 \mathrm{ab}$ & $93.67 \mathrm{a}$ & $101.7 \mathrm{ab}$ & $103.7 \mathrm{ab}$ \\
& $\mathrm{V}_{3}$ & $68.00 \mathrm{bc}$ & $102.0 \mathrm{a}$ & $102.30 \mathrm{a}$ & $97.67 \mathrm{abc}$ & $99.67 \mathrm{ab}$ \\
& $\mathrm{V}_{1}$ & $69.67 \mathrm{~b}$ & $86.67 \mathrm{~b}$ & $91 \mathrm{ab}$ & $88.67 \mathrm{~d}$ & $90.67 \mathrm{~cd}$ \\
$\mathrm{~F}_{3}$ & $\mathrm{~V}_{2}$ & $61.33 \mathrm{c}$ & $92.67 \mathrm{ab}$ & $99.33 \mathrm{a}$ & $94.00 \mathrm{~b}-\mathrm{d}$ & $96.33 \mathrm{bc}$ \\
& $\mathrm{V}_{3}$ & $64.33 \mathrm{bc}$ & $92.67 \mathrm{ab}$ & $105.30 \mathrm{a}$ & $100.00 \mathrm{a}-\mathrm{c}$ & $104.00 \mathrm{ab}$ \\
\hline \multicolumn{2}{l}{ Level of significance } & $* *$ & $*$ & $*$ & $* *$ & $* *$ \\
\hline \multicolumn{2}{l}{$\mathrm{CV}(\%)$} & 5.00 & 7.99 & 11.10 & 5.82 & 5.49 \\
\hline
\end{tabular}

In a column, means followed by a different letter(s) differed significantly by Duncan's Multiple Range Test at $P \leq 5 \%$ level of probability.

NS indicates insignificant, *indicates significant at $5 \%$ level of probability

**indicates significant at $1 \%$ level of probability

Table 2. Effect of fertilization on leaf number of different rice varieties at different stages

\begin{tabular}{|c|c|c|c|c|c|}
\hline \multirow{2}{*}{\multicolumn{2}{|c|}{$\begin{array}{l}\text { Treatments } \\
\text { Varieties }\end{array}$}} & \multicolumn{4}{|c|}{ Leaf number } \\
\hline & & 30 DAT & Vegetative & Booting & Anthesis \\
\hline \multicolumn{2}{|c|}{$V_{1=B i n a d h a n}-7$} & 67.56 & $85.56 \mathrm{ab}$ & 90.11 & 54.56 \\
\hline \multicolumn{2}{|c|}{$V_{2=}$ BRRI dhan56 } & 69.56 & $94.33 a$ & 93.11 & 53.89 \\
\hline \multicolumn{2}{|c|}{$\mathrm{V}_{3=} \mathrm{BRRI}$ dhan66 } & 80.89 & $75.56 b$ & 91.56 & 51.67 \\
\hline \multicolumn{2}{|c|}{ Level of significance } & NS & ${ }^{*}$ & NS & NS \\
\hline \multicolumn{6}{|c|}{ Fertilization } \\
\hline \multicolumn{2}{|c|}{$\mathrm{F}_{1}=$ Inorganic } & 77.11 & $90.22 \mathrm{ab}$ & $95.56 a$ & 57.00 \\
\hline \multicolumn{2}{|c|}{$\mathrm{F}_{2}=$ Compost 20 tha $^{-1}$} & 63.56 & $70.11 b$ & $81.78 a$ & 49.67 \\
\hline \multicolumn{2}{|c|}{$\mathrm{F}_{3}=$ Compost 30 tha $^{-1}$} & 77.33 & $95.11 \mathrm{a}$ & $97.44 a$ & 53.44 \\
\hline \multicolumn{2}{|c|}{ Level of significance } & NS & ${ }^{*}$ & NS & NS \\
\hline \multicolumn{6}{|c|}{ Interaction } \\
\hline \multirow{4}{*}{$\mathrm{F}_{1}$} & $\mathrm{~V}_{1}$ & 80.00 & 98.00 & 90.67 & 58.67 \\
\hline & $\mathrm{V}_{2}$ & 54.67 & 68.33 & 89.33 & 54.67 \\
\hline & $\mathrm{V}_{3}$ & 68.00 & 90.33 & 90.33 & 50.33 \\
\hline & $\mathrm{V}_{1}$ & 77.67 & 104.0 & 100.7 & 58.33 \\
\hline \multirow[t]{3}{*}{$\mathrm{F}_{2}$} & $\mathrm{~V}_{2}$ & 59.00 & 76.67 & 78.67 & 45.67 \\
\hline & $\mathrm{V}_{3}$ & 72.00 & 102.3 & 100.00 & 57.67 \\
\hline & $\mathrm{V}_{1}$ & 73.67 & 68.67 & 95.33 & 54.00 \\
\hline \multirow[t]{2}{*}{$\mathrm{F}_{3}$} & $\mathrm{~V}_{2}$ & 77.00 & 65.33 & 77.33 & 48.67 \\
\hline & $\mathrm{V}_{3}$ & 92.00 & 92.67 & 102.00 & 52.33 \\
\hline \multicolumn{2}{|c|}{ Level of significance } & NS & NS & NS & NS \\
\hline \multicolumn{2}{|c|}{ CV (\%) } & 28.98 & 23.32 & 17.94 & 15.78 \\
\hline
\end{tabular}

In a column, means followed by a different letter(s) differed significantly by Duncan's Multiple Range Test at $P \leq 5 \%$ level of probability.

NS indicates insignificant

*indicates significant at $5 \%$ level of probability

**indicates significant at $1 \%$ level of probability 


\section{Number of effective tillers hill ${ }^{-1}$}

The effects of varieties on the number of effective tillers hill ${ }^{-1}$ at different days after transplanting (DAT) were statistically significant (Figure 1). Results showed that the number of effective tiller hill $^{-1}$ increased with various doses of poultry-based compost. The highest number of effective tiller hill ${ }^{-1}$ was recorded from $\mathrm{V}_{2}$ (BRRI dhan56) (11.44 and 12.33 at anthesis and maturity stage, respectively). The lowest number of effective tillers hill ${ }^{-1}$ was recorded from $V_{3}$ (BRRI dhan66) (10.89 and 11.56 at anthesis and maturity stage, respectively). The number of effective tillers hill ${ }^{-1}$ was influenced by different fertilization presented in Fig. 2 . It was observed that $F_{1}$ (inorganic) produced the highest number of effective tillers hill-1 ${ }^{-1}$ (11.89 at anthesis stage), and $\mathrm{F}_{2}$ (compost-20 t ha ${ }^{-1}$ ) showed the lowest result (12.33 at maturity stage). The number of effective tillers hill ${ }^{-1}$ was significantly affected by the interaction between varieties and fertilization (Fig. 3). Results indicated that the highest number of effective tillers hill ${ }^{-1}(12.67,13.67)$ was found in the combination of $\mathrm{V}_{1} \mathrm{~F}_{2}$ (Binadhan-7 with compost-20 t ha-1). Nitrogen availability may play an important role in the tiller generation. Inorganic fertilizer is a good source of available nitrogen with flooding irrigation as per Islam et al. (2014), Shaha (2014) and Hossain (2010). Alam et al. (2012) also stated similar findings.

Table 3. Effect of fertilization on tiller number of different rice varieties at different stages

\begin{tabular}{|c|c|c|c|c|c|c|}
\hline \multirow{2}{*}{\multicolumn{2}{|c|}{$\begin{array}{l}\text { Treatments } \\
\text { Varieties }\end{array}$}} & \multicolumn{5}{|c|}{ Tiller number } \\
\hline & & 30DAT & Vegetative & Booting & Anthesis & Maturity \\
\hline \multicolumn{2}{|c|}{$V_{1=\text { Binadhan }-7}$} & 13.00 & 13.44 & 14.89 & 12.78 & 13.44 \\
\hline \multicolumn{2}{|c|}{$\mathrm{V}_{2=}$ BRRI dhan56 } & 15.33 & 14.89 & 15.56 & 13.11 & 14.11 \\
\hline \multicolumn{2}{|c|}{$\mathrm{V}_{3=}$ BRRI dhan 66} & 13.67 & 13.22 & 15.56 & 12.11 & 12.78 \\
\hline \multicolumn{2}{|c|}{ Level of significance } & Ns & NS & NS & NS & NS \\
\hline \multicolumn{7}{|c|}{ Fertilization } \\
\hline \multicolumn{2}{|c|}{$\mathrm{F}_{1}=$ Inorganic } & 15.33 & 15.33 & 15.89 & 13.67 & 15.44 \\
\hline \multicolumn{2}{|c|}{$\mathrm{F}_{2}=$ Compost 20 tha $^{-1}$} & 12.56 & 11.89 & 13.89 & 12.56 & 12.56 \\
\hline \multicolumn{2}{|c|}{$\mathrm{F}_{3}=$ Compost 30 tha $^{-1}$} & 14.11 & 14.33 & 16.22 & 11.78 & 12.33 \\
\hline \multicolumn{2}{|c|}{ Level of significance } & Ns & $*$ & NS & NS & $* *$ \\
\hline \multicolumn{7}{|c|}{ Interaction } \\
\hline \multirow{3}{*}{$\mathrm{F}_{1}$} & $\mathrm{~V}_{1}$ & $17.00 \mathrm{a}$ & 16.33 & 14.67 & 14.00 & $15.33 \mathrm{ab}$ \\
\hline & $\mathrm{V}_{2}$ & $8.33 b$ & 11.67 & 15.00 & 13.00 & $13.33 \mathrm{abc}$ \\
\hline & $\mathrm{V}_{3}$ & 13.67ab & 12.33 & 15.00 & 11.33 & $11.67 \mathrm{c}$ \\
\hline \multirow{3}{*}{$\mathrm{F}_{2}$} & $\mathrm{~V}_{1}$ & $16.67 a$ & 17.33 & 17.00 & 14.00 & $16 a$ \\
\hline & $\mathrm{V}_{2}$ & $15.67 \mathrm{a}$ & 12.00 & 13.00 & 13.00 & $12.67 b c$ \\
\hline & $\mathrm{V}_{3}$ & $13.67 \mathrm{ab}$ & 15.33 & 16.67 & 12.33 & $13.67 \mathrm{abc}$ \\
\hline \multirow{3}{*}{$\mathrm{F}_{3}$} & $\mathrm{~V}_{1}$ & $12.33 \mathrm{ab}$ & 12.33 & 16.00 & 13.00 & $15 \mathrm{ab}$ \\
\hline & $\mathrm{V}_{2}$ & $13.67 \mathrm{ab}$ & 12.00 & 13.67 & 11.67 & $11.67 \mathrm{c}$ \\
\hline & $\mathrm{V}_{3}$ & $15.00 \mathrm{a}$ & 15.33 & 17.00 & 11.67 & $11.67 \mathrm{c}$ \\
\hline \multicolumn{2}{|c|}{ Level of significance } & $*$ & NS & NS & NS & $* * *$ \\
\hline \multicolumn{2}{|c|}{ CV (\%) } & 18.49 & 21.70 & 20.16 & 17.52 & 15.75 \\
\hline
\end{tabular}

In a column, means followed by different letter(s) differed significantly by Duncan's Multiple Range Test at $\mathrm{P}$ $\leq 5 \%$ level of probability.

NS indicates insignificant

*indicates significant at $5 \%$ level of probability

**indicates significant at $1 \%$ level of probability 


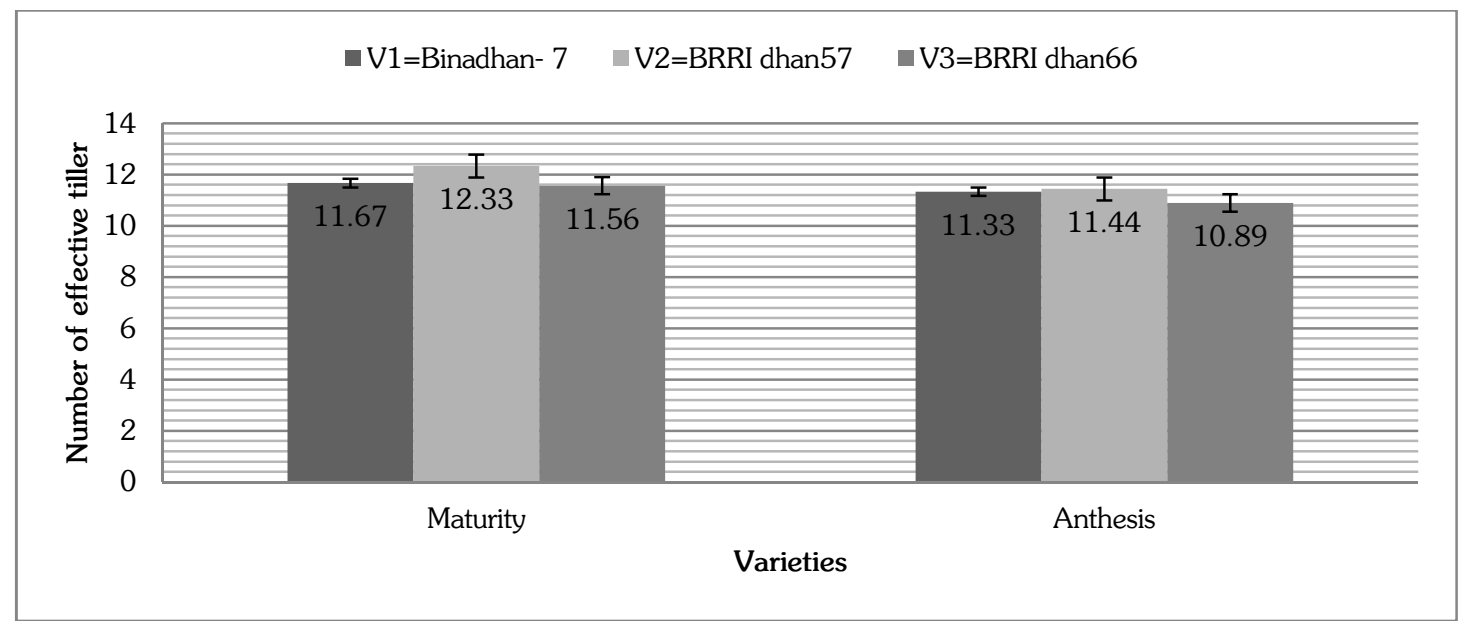

Fig. 1. Effect of different varieties on effective tiller/hill at anthesis and maturity stages

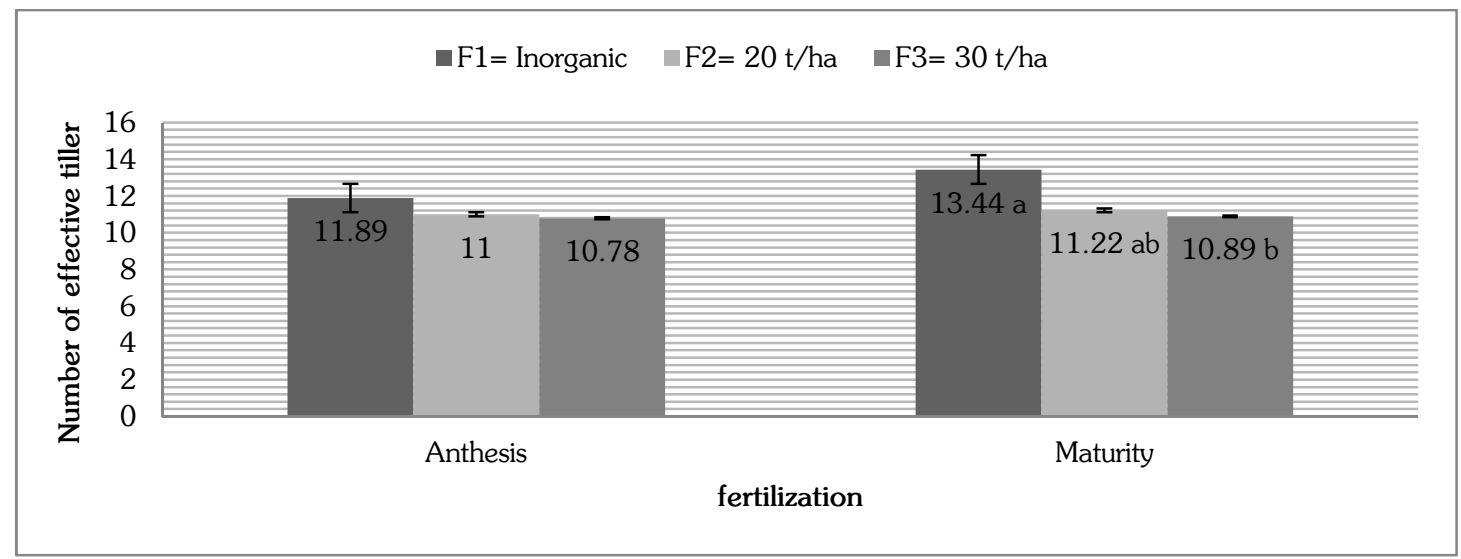

Fig. 2. Effect of different fertilization on effective tiller/hill in rice at anthesis and maturity stages

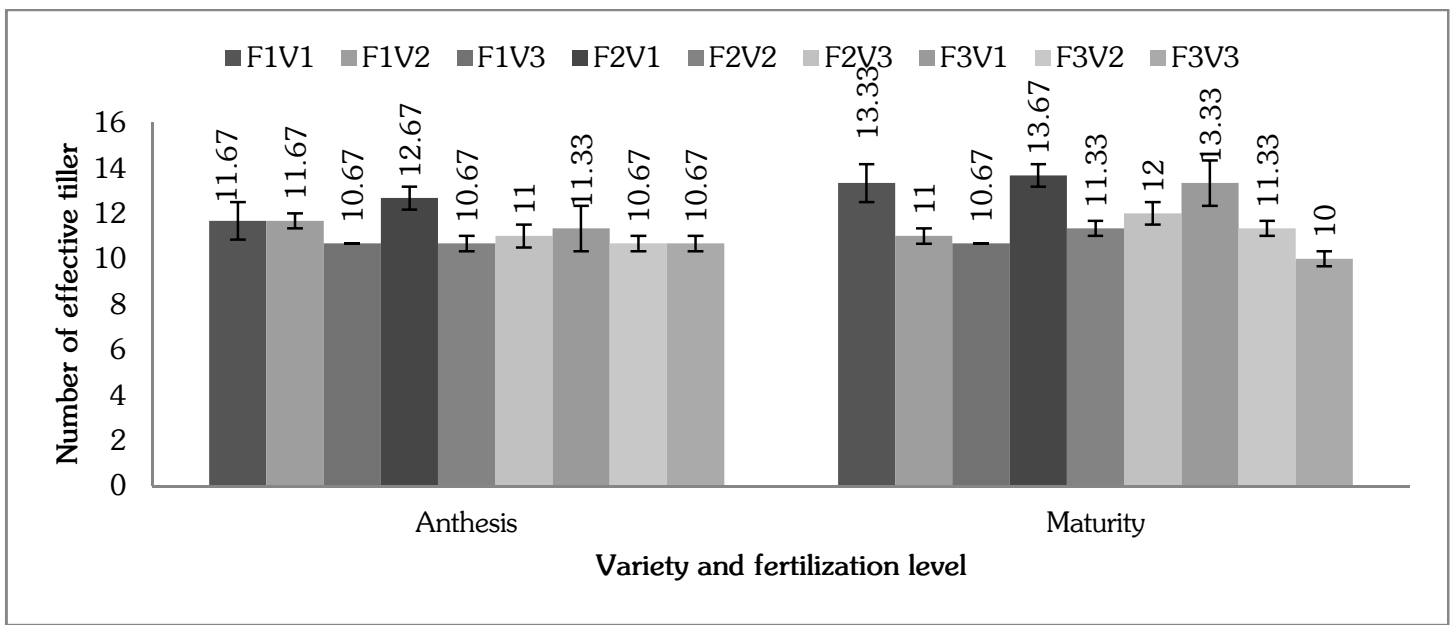

Fig.3. Interaction effect between different varieties and different fertilization on effective tiller/hill in rice at anthesis and maturity stages 


\section{Stem dry weight hill-1}

Stem dry weight per plant was affected by the interaction between variety and fertilization presented in Table 4 . At the maximum vegetative stage, the highest stem dry weight per plant (9.93g) was obtained in $\mathrm{V}_{3} \mathrm{~F}_{2}$ (BRRI dhan66 with compost-20 $\mathrm{tha}^{-1}$ ), and the lowest stem dry weight plant ${ }^{-1}(6.2 \mathrm{~g})$ was obtained in $\mathrm{V}_{3} \mathrm{~F}_{1}$ (BRRI dhan66 with inorganic). At the booting stage, the highest stem dry weight plant ${ }^{-1}(15.29 \mathrm{~g})$ was obtained in $\mathrm{V}_{3} \mathrm{~F}_{3}$ (BRRI dhan66 with compost$30 \mathrm{t} \mathrm{ha}^{-1}$ ) and the lowest stem dry weight plant ${ }^{-1}\left(10.30 \mathrm{~g}\right.$ ) was obtained in $\mathrm{V}_{2} \mathrm{~F}_{2}$ (BRRI dhan56 with compost-20 $\left.\mathrm{t} \mathrm{ha}^{-1}\right)$. At the anthesis stage, the highest stem dry weight plant ${ }^{-1}(42.14 \mathrm{~g})$ was obtained in $\mathrm{V}_{1} \mathrm{~F}_{2}$ (Binadhan-7 with compost-20 t ha-1), which was statistically similar in $\mathrm{V}_{1} \mathrm{~F}_{1}$ (Binadhan-7 with inorganic) (42.02 g), and the lowest stem dry weight plant ${ }^{-1}(27.65 \mathrm{~g})$ was recorded from $\mathrm{V}_{2} \mathrm{~F}_{3}$ (BRRI dhan56 with compost-30 $\mathrm{tha}^{-1}$ ).

\section{Leaf dry weight hill ${ }^{-1}$}

Leaf dry weight plant ${ }^{-1}$ was affected by the interaction between variety and fertilization presented in Table 4. At the vegetative stage, the highest leaf dry weight plant ${ }^{-1}(8.26 \mathrm{~g})$ was obtained in $\mathrm{V}_{3} \mathrm{~F}_{2}$ (BRRI dhan66 with compost-20 $\mathrm{t} \mathrm{ha}^{-1}$ ) and the lowest leaf dry weight plant ${ }^{-1}(5.50 \mathrm{~g}$ ) was obtained in $\mathrm{V}_{1} \mathrm{~F}_{3}$ (Binadhan-7 with compost-30 tha-1). At the booting stage, the highest leaf dry weight plant ${ }^{-1}(9.86 \mathrm{~g})$ was obtained in $\mathrm{V}_{3} \mathrm{~F}_{3}$ (BRRI dhan66 with compost-30 $\mathrm{t} \mathrm{ha}^{-1}$ ) and the lowest leaf dry weight plant ${ }^{-1}\left(6.65 \mathrm{~g}\right.$ ) was obtained in $\mathrm{V}_{2} \mathrm{~F}_{3}$ (BRRI dhan56 with compost-30 t ha-1).

Table 4. Effect of fertilization on stem dry weight and leaf dry weight of different ricevarieties at different stages

\begin{tabular}{|c|c|c|c|c|c|c|c|}
\hline \multirow{2}{*}{\multicolumn{2}{|c|}{$\begin{array}{l}\text { Treatments } \\
\text { Varieties }\end{array}$}} & \multicolumn{3}{|c|}{ Stem dry weight (g) } & \multicolumn{3}{|c|}{ Leaf dry weight (g) } \\
\hline & & Vegetative & Booting & Anthesis & Vegetative & Booting & Anthesis \\
\hline \multicolumn{2}{|c|}{$\mathrm{V}_{1=\text { Binadhan-7 }}$} & 7.44 & 11.93 & 37.28 & 6.91 & 8.18 & 4.66 \\
\hline \multicolumn{2}{|c|}{$\mathrm{V}_{2=} \mathrm{BRRI}$ dhan 56} & 9.24 & 13.17 & 37.29 & 7.52 & 8.18 & 4.52 \\
\hline \multicolumn{2}{|c|}{$\mathrm{V}_{3=}$ BRRI dhan 66} & 7.38 & 13.29 & 34.87 & 6.22 & 8.23 & 4.33 \\
\hline \multicolumn{2}{|c|}{ Level of significance } & NS & NS & NS & NS & NS & NS \\
\hline \multicolumn{8}{|c|}{ Fertilization } \\
\hline \multirow{4}{*}{\multicolumn{2}{|c|}{$\begin{array}{l}\mathrm{F}_{1}=\text { Inorganic } \\
\mathrm{F}_{2}=\text { Compost } 20 \text { tha }^{-1} \\
\mathrm{~F}_{3}=\text { Compost } 30 \text { tha }^{-1} \\
\text { Level of significance }\end{array}$}} & 9.00 & 13.18 & $41.37 a$ & 7.28 & 8.44 & 4.91 \\
\hline & & 6.62 & 11.47 & $30.57 b$ & 5.73 & 7.17 & 4.13 \\
\hline & & 8.44 & 13.74 & $37.50 \mathrm{a}$ & 7.64 & 8.98 & 4.47 \\
\hline & & NS & NS & $* *$ & NS & NS & NS \\
\hline \multicolumn{8}{|c|}{ Interaction } \\
\hline \multirow{3}{*}{$\mathrm{F}_{1}$} & $\mathrm{~V}_{1}$ & 9.60 & 12.27 & $42.02 a$ & 8.12 & 8.23 & 4.81 \\
\hline & $\mathrm{V}_{2}$ & 6.52 & 12.33 & $33.95 a-c$ & 5.63 & 7.80 & 4.91 \\
\hline & $\mathrm{V}_{3}$ & 6.20 & 11.20 & $35.87 a-c$ & 7.00 & 8.51 & 4.27 \\
\hline \multirow{3}{*}{$\mathrm{F}_{2}$} & $\mathrm{~V}_{1}$ & 10.83 & 14.47 & $42.14 a$ & 8.23 & 8.90 & 5.26 \\
\hline & $\mathrm{V}_{2}$ & 6.96 & 10.30 & $30.09 b c$ & 6.06 & 7.08 & 3.70 \\
\hline & $\mathrm{V}_{3}$ & 9.93 & 14.73 & 39.63a & 8.26 & 8.57 & 4.61 \\
\hline \multirow{3}{*}{$\mathrm{F}_{3}$} & $\mathrm{~V}_{1}$ & 6.56 & 12.80 & $39.96 a$ & 5.50 & 8.19 & 4.66 \\
\hline & $\mathrm{V}_{2}$ & 6.40 & 11.77 & $27.65 c$ & 5.50 & 6.65 & 3.80 \\
\hline & $\mathrm{V}_{3}$ & 9.19 & 15.29 & $37 a b$ & 7.66 & 9.86 & 4.52 \\
\hline \multicolumn{2}{|c|}{ Level of significance } & NS & NS & *** & NS & NS & NS \\
\hline \multicolumn{2}{|c|}{ CV (\%) } & 23.83 & 23.35 & 16.31 & 28.18 & 22.20 & 19.13 \\
\hline
\end{tabular}

In a column, means followed by a different letter(s) differed significantly by Duncan's Multiple Range Test at $P \leq 5 \%$ level of probability.

NS indicates insignificant

*indicates significant at $5 \%$ level of probability

**indicates significant at $1 \%$ level of probability 
At the anthesis stage, the highest leaf dry weight plant ${ }^{-1}(5.26 \mathrm{~g})$ was obtained in $\mathrm{V}_{1} \mathrm{~F}_{2}$ (Binadhan-7 with compost-20 $\mathrm{t} \mathrm{ha}^{-1}$ ), and the lowest leaf dry weight plant ${ }^{-1}$ (3.7 g) was recorded from $\mathrm{V}_{2} \mathrm{~F}_{2}$ (BRRI dhan56 with compost-20 $\mathrm{t} \mathrm{ha}^{-1}$ ).

\section{Root dry weight hill ${ }^{-1}$}

Root dry weight plant ${ }^{-1}$ was affected by the interaction between variety and fertilization presented in Table 5. At the vegetative stage, the highest root dry weight plant ${ }^{-1}(4.3 \mathrm{~g})$ was obtained in $\mathrm{V}_{3} \mathrm{~F}_{2}$ (BRRI dhan66 with compost-20 t ha-1), and the lowest root dry weight plant ${ }^{-1}(2.18 \mathrm{~g})$ was obtained in $\mathrm{V}_{2} \mathrm{~F}_{2}$ (BRRI dhan56 with compost-20 tha-1). At the booting stage, the highest root dry weight plant ${ }^{-1}(3.86 \mathrm{~g})$ was obtained in $\mathrm{V}_{2} \mathrm{~F}_{1}$ (BRRI dhan56 with inorganic), and the lowest root dry weight plant ${ }^{-1}(2.65 \mathrm{~g})$ was obtained in $\mathrm{V}_{3} \mathrm{~F}_{2}$ (BRRI dhan66 with compost-20 t ha-1).At the anthesis stage, the highest root dry weight plant ${ }^{-1}(3.01 \mathrm{~g})$ was obtained in $\mathrm{V}_{1} \mathrm{~F}_{1}$ Binadhan-7 with inorganic), and the lowest root dry plant ${ }^{-1}(2.2 \mathrm{~g})$ was recorded from $\mathrm{V}_{2} \mathrm{~F}_{3}$ (BRRI dhan56 with compost-30 $\mathrm{t} \mathrm{ha}^{-1}$ ).

\section{Leaf area}

Leaf area evaluated for different varieties and fertilization is presented in Table 5. Leaf area was found with almost similar in each treatment. The maximum leaf area was recorded from $V_{2}$ (BRRI dhan56) (5588 $\mathrm{cm}^{2}$ and $5229 \mathrm{~cm}^{2}$ at vegetative and booting stages, respectively) and the maximum leaf area was recorded from $V_{1}$ (Binadhan-7) $\left(3126 \mathrm{~cm}^{2}\right.$ and $3159 \mathrm{~cm}^{2}$ at anthesis and maturity stages, respectively).

Table 5. Effect of fertilization on root dry weight and leaf area of different rice varieties at different stages

\begin{tabular}{|c|c|c|c|c|c|c|c|c|}
\hline \multicolumn{2}{|c|}{ Treatments } & \multicolumn{3}{|c|}{ Root dry weight (g) } & \multicolumn{4}{|c|}{ Leaf area $\left(\mathrm{cm}^{2}\right)$} \\
\hline \multicolumn{2}{|c|}{ Varieties } & Vegetative & Booting & Anthesis & Vegetative & Booting & Anthesis & Maturity \\
\hline \multicolumn{2}{|c|}{$\mathrm{V}_{1=\text { Binadhan-7 }}$} & 2.79 & $3.05^{5}$ & 2.67 & 5028 & 5151 & 3126 & 3159 \\
\hline \multicolumn{2}{|c|}{$\mathrm{V}_{2=} \mathrm{BRRI}$ dhan 56} & 3.29 & 3.29 & 2.61 & 5588 & 5229 & 2805 & 3119 \\
\hline \multicolumn{2}{|c|}{$\mathrm{V}_{3=} \mathrm{BRRI}$ dhan66 } & 2.92 & 3.35 & 2.46 & 4500 & 5210 & 2905 & 3116 \\
\hline \multicolumn{2}{|c|}{ Level of significance } & NS & NS & NS & NS & NS & NS & NS \\
\hline \multicolumn{9}{|c|}{ Fertilization } \\
\hline \multicolumn{2}{|c|}{$\mathrm{F}_{1}=$ Inorganic } & 3.08 & 3.13 & 2.78 & 5305 & 5352 & 3096 & 3156 \\
\hline \multicolumn{2}{|c|}{$\mathrm{F}_{2}=$ Compost 20 tha $^{-1}$} & 2.59 & 2.92 & 2.32 & 4227 & 4628 & 2675 & 3058 \\
\hline \multicolumn{2}{|c|}{$\mathrm{F}_{3}=$ Compost 30 tha $^{-1}$} & 3.34 & 3.65 & 2.64 & 5585 & 5610 & 3065 & 3181 \\
\hline \multicolumn{2}{|c|}{ Level of significance } & NS & NS & NS & NS & NS & NS & NS \\
\hline \multicolumn{9}{|c|}{ Interaction } \\
\hline \multirow{3}{*}{$\mathrm{F}_{1}$} & $\mathrm{~V}_{1}$ & 2.93 & 2.82 & 3.01 & 5755 & 5169 & $3297 a$ & 2924 \\
\hline & $\mathrm{V}_{2}$ & 2.66 & 3.86 & 2.52 & 4163 & 5135 & $3082 \mathrm{ab}$ & 3458 \\
\hline & $\mathrm{V}_{3}$ & 2.80 & 2.89 & 2.48 & 5167 & 5150 & 2997ab & 3096 \\
\hline \multirow{3}{*}{$\mathrm{F}_{2}$} & $\mathrm{~V}_{1}$ & 3.40 & 3.45 & 2.76 & 6095 & 5557 & 2989ab & 3356 \\
\hline & $\mathrm{V}_{2}$ & 2.18 & 3.58 & 2.25 & 4667 & 4356 & $2338 b$ & 2634 \\
\hline & $\mathrm{V}_{3}$ & 4.30 & 2.65 & 2.84 & 6003 & 5774 & 3089ab & 3367 \\
\hline \multirow{3}{*}{$\mathrm{F}_{3}$} & $\mathrm{~V}_{1}$ & 2.90 & 3.65 & 2.58 & 4065 & 5331 & 3003ab & 3188 \\
\hline & $\mathrm{V}_{2}$ & 2.93 & 2.98 & 2.20 & 3850 & 4392 & 2603ab & 3080 \\
\hline & $\mathrm{V}_{3}$ & 2.93 & 3.22 & 2.60 & 5584 & 5908 & 3109ab & 3081 \\
\hline \multicolumn{2}{|c|}{ Level of significance } & NS & NS & NS & NS & NS & $*$ & NS \\
\hline \multicolumn{2}{|c|}{ CV (\%) } & 21.69 & 21.44 & 22.90 & 23.83 & 18.06 & 18.80 & 17.18 \\
\hline
\end{tabular}

In a column, means followed by a different letter(s) differed significantly by Duncan's Multiple Range Test at $P \leq 5 \%$ level of probability.

NS indicates insignificant

*indicates significant at $5 \%$ level of probability

**indicates significant at $1 \%$ level of probability 
The maximum leaf area was recorded from $\mathrm{F}_{3}$ (compost-30 $\left.\mathrm{t} \mathrm{ha}^{-1}\right)\left(5585 \mathrm{~cm}^{2}, 5610 \mathrm{~cm}^{2}\right.$ and 3181 at vegetative, booting and maturity stages, respectively), and the maximum leaf area was recorded from $\mathrm{F}_{1}$ (Inorganic) (3096 $\mathrm{cm}^{2}$ at anthesis stage). At the vegetative stage, the highest leaf area $\left(6095 \mathrm{~cm}^{2}\right)$ was obtained in $\mathrm{V}_{3} \mathrm{~F}_{2}$ (BRRI 66 with compost-20 $\mathrm{t} \mathrm{ha}{ }^{-1}$ ) and the lowest leaf area $\left(3850 \mathrm{~cm}^{2}\right)$ was obtained in $\mathrm{V}_{2} \mathrm{~F}_{3}$ (BRRI dhan56 with compost-30 $\mathrm{t} \mathrm{ha}{ }^{-1}$ ). At the booting stage, the highest leaf area $\left(5908 \mathrm{~cm}^{2}\right)$ was obtained in $\mathrm{V}_{3} \mathrm{~F}_{3}$ (BRRI dhan66 with compost-30 $\mathrm{t}$ $\mathrm{ha}^{-1}$ ), and the lowest leaf area $\left(4356 \mathrm{~cm}^{2}\right.$ ) was obtained in $\mathrm{V}_{2} \mathrm{~F}_{2}$ (BRRI dhan56 with compost-20 $\left.t \mathrm{ha}^{-1}\right)$. At the anthesis stage, the highest leaf area $\left(3109 \mathrm{~cm}^{2}\right)$ was obtained in $\mathrm{V}_{3} \mathrm{~F}_{3}$ (BRRI dhan66 with compost-30 $\left.\mathrm{t} \mathrm{ha}^{-1}\right)$ and the lowest leaf area $\left(2338 \mathrm{~cm}^{2}\right)$ was obtained in $\mathrm{V}_{2} \mathrm{~F}_{2}$ (BRRI dhan56 with compost-20 ton ha ${ }^{-1}$ ). At the maturity stage, the highest leaf area (3458 $\mathrm{cm}^{2}$ ) was obtained in $\mathrm{V}_{2} \mathrm{~F}_{1}$ (BRRI 56 with inorganic), and the lowest leaf area $\left(2634 \mathrm{~cm}^{2}\right)$ was obtained in $\mathrm{V}_{2} \mathrm{~F}_{2}$ (BRRI dhan56 with compost-20 $\mathrm{t} \mathrm{ha}^{-1}$ ).

\section{SPAD value}

SPAD value was significantly influenced by poultry litter based composts, but the SPAD value was not significantly influenced in the case of varieties (Table 6). Result revealed that SPAD value was increased with the use of composts. The highest SPAD value (48.74) was recorded from $\mathrm{F}_{3}$ and the lowest SPAD value (44.77) from $\mathrm{F}_{2}$ at vegetative stages in other stages SPAD value was not significant. The interaction effect of fertilization and different varieties was significantly influenced by SPAD value at vegetative and booting stages.

Table 6. Effect of fertilization on SPAD value of different rice varieties at different stages

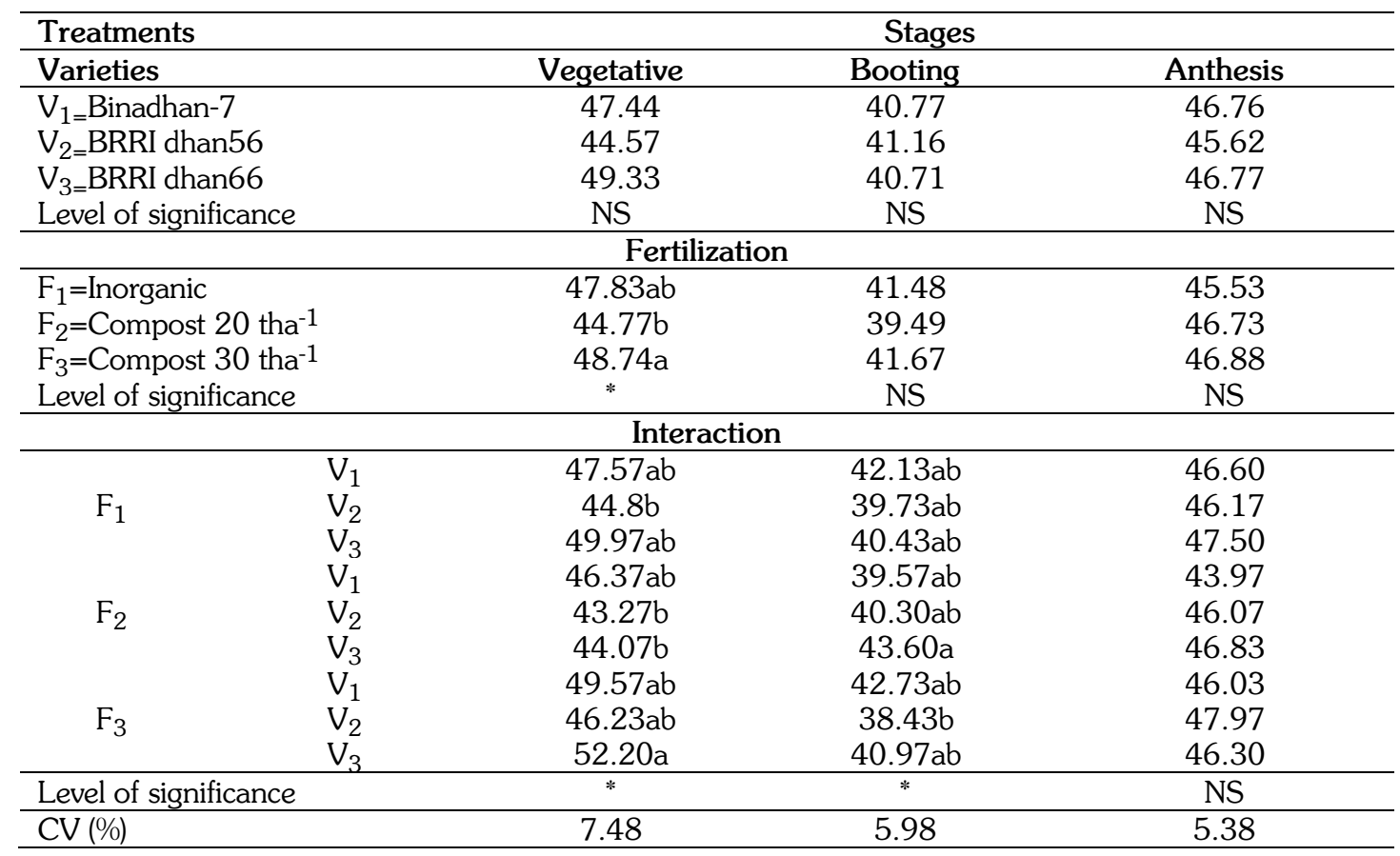

In a column, means followed by a different letter(s) differed significantly by Duncan's Multiple Range Test at $P \leq 5 \%$ level of probability.

NS indicates insignificant

*indicates significant at 5\% level of probability

$* *$ indicates significant at $1 \%$ level of probability 
The highest SPAD value (52.20) at the vegetative stage was recorded from $\mathrm{F}_{3} \mathrm{~V}_{3}$ (compost $-30 \mathrm{t}$ $\mathrm{ha}^{-1}$ with BRRI dhan66), and the lowest SPAD value (43.27) was recorded from $\mathrm{F}_{2} \mathrm{~V}_{2}$. The highest SPAD value (43.60) at the booting stage was recorded from $\mathrm{F}_{2} \mathrm{~V}_{3}$. The lowest $\mathrm{SPAD}$ value (38.43) was recorded from $\mathrm{F}_{3} \mathrm{~V}_{2}$ at the booting stage. SPAD value was significantly influenced by poultry litter based composts but the SPAD value insignificant in the case of varieties (Table 8). Result revealed that SPAD value was increased with the use of composts. The highest SPAD value (48.74) was recorded from $\mathrm{F}_{3}$ and the lowest SPAD value (44.77) from $\mathrm{F}_{2}$ at the vegetative stages, whereas in other stages SPAD value was not significant.

The interaction effect of fertilization and different varieties was significantly influenced by SPAD value at vegetative and booting stages. The highest SPAD value (52.20) at the vegetative stage was recorded from $F_{3} V_{3}$ whreas the lowest SPAD value (43.27) was recorded from $F_{2} V_{2}$ at the vegetative stage. The highest SPAD value (43.60) at the booting stage was recorded from $\mathrm{F}_{2} \mathrm{~V}_{3}$ and the lowest SPAD value (38.43) from $\mathrm{F}_{3} \mathrm{~V}_{2}$ at the booting stage. Zubaer et al. (2007) and Chowdhury et al. (2020) found a similar result at drought conditions that compost and nutrients increased chlorophyll contents in a crop plant.

\section{Panicle length}

Panicle length was not significantly influenced by poultry litter based composts but significantly influenced by different rice varieties (Table 7). The longest panicle length $(22.9 \mathrm{~cm})$ was produced by $V_{1}$ (Binadhan-7), and the shortest panicle length $(20.48 \mathrm{~cm})$ produced by $V_{3}$ (var. BRRI dhan66). Panicle length was not significantly influenced by poultry litter based composts (Table 9). Interaction between fertilizer and different rice varieties significantly influenced the panicle length. The longest panicle $(24.16 \mathrm{~cm})$ found in $\mathrm{F}_{1} \mathrm{~V}_{1}$ (inorganic with Binadhan-7) treatment and the shortest one $(19.83 \mathrm{~cm})$ found in $\mathrm{F}_{3} \mathrm{~V}_{3}$ (compost-30 $\mathrm{t} \mathrm{ha}^{-1}$ with BRRI dhan66). Sarker et al. (2013) and Haque (2013) was found similar results of increased panicle length due to the application of manure on rice plants.

\section{0-grain weight}

Production of rice yield also depends on 1000-grain weight, which was significantly influenced by poultry litter based composts, but the 1000-grain weight was not significantly influenced by varieties (Table 7). Results revealed that the highest 1000 -grain weight $(22.23 \mathrm{~g})$ was recorded from $F_{3}$ and the lowest 1000 -grain weight $(19.66 \mathrm{~g})$ was recorded from $F_{2}$. This result was in agreement with the reports of Aziz (2008).

The interaction effect of fertilizer and different varieties significantly influenced the 1000-grain weight. The highest 1000 -grain weight $(23.57 \mathrm{~g})$ was recorded from $\mathrm{F}_{3} \mathrm{~V}_{3}$ treatment and the lowest 1000 -grain weight (19.27 g) was recorded from $\mathrm{F}_{1} \mathrm{~V}_{2}$ (inorganic with BRRI dhan56).

\section{Grain yield}

Grain yield was significantly influenced by poultry litter based composts, but the grain yield (g pot $^{-1}$ ) was not significantly influenced in the case of varieties (Table 7). Result revealed that grain yield was increased with the use of composts. The highest grain yield $\left(23.61 \mathrm{~g} \mathrm{pot}^{-1}\right)$ was recorded from $F_{1}$ (inorganic). The lowest grain yield (14.32 $\left.\mathrm{g} \mathrm{pot}^{-1}\right)$ was recorded from $F_{2}$ (compost-2 $\mathrm{t} \mathrm{ha}{ }^{-1}$ ). The interaction effect of fertilizer and different varieties was significantly influenced by grain yield. The highest grain yield $\left(24.58 \mathrm{~g} \mathrm{pot}^{-1}\right)$ was recorded from $F_{3} V_{1}$ (compost-30 $\mathrm{t} \mathrm{ha}^{-1}$ with Binadhan-7). The lowest grain yield (11.98 $\mathrm{g} \mathrm{pot}^{-1}$ ) was recorded from $\mathrm{F}_{2} \mathrm{~V}_{2}$ (compost $-20 \mathrm{t} \mathrm{ha}^{-1}$ with BRRI dhan56).Islam et al. (2013) and Najafi and Abbasi (2013) also found a similar result in rice production. Saleque et al. (2004) reported that the application of compost increased the yield of rice. 


\section{Straw yield}

The straw yield was significantly influenced by poultry litter based composts (Table 7). Result revealed that straw yield was increased with the use of composts. But the straw yield was not significantly influenced in varieties. The highest straw yield $(18.19 \mathrm{~g})$ was recorded from $\mathrm{F}_{1}$ (inorganic). The lowest straw yield $(14.76 \mathrm{~g})$ was recorded from $F_{3}$. The combined effect of compost and different varieties significantly influenced the straw yield. The highest straw yield $(18.6 \mathrm{~g})$ was recorded from $\mathrm{F}_{2} \mathrm{~V}_{1}$ (compost $20 \mathrm{t} \mathrm{ha}^{-1}$ with Binadhan-7). The lowest straw yield $(13.5 \mathrm{~g})$ was recorded from $\mathrm{F}_{3} \mathrm{~V}_{3}$ (compost-30 t ha ${ }^{-1}$ with BRRI dhan66) treatment.

\section{Harvest index}

As a useful index of measuring the plant biomass converted into fruitful economic yield, the harvest index was significantly influenced by poultry litter based composts (Table 7). The harvest index was not significantly influenced by varieties. Results revealed that the harvest index was increased with the use of different composts treatment. The highest harvest index (58.2\%) was recorded from $\mathrm{F}_{3}$ (compost $30 \mathrm{tha}^{-1}$ ). The lowest harvest index $(47.89 \%)$ was recorded from $\mathrm{F}_{2}$ (compost-20 t ha-1). The combined effect of poultry litter based composts and different varieties significantly influenced in harvest index. The highest harvest index (62.78\%) was recorded from $\mathrm{F}_{1} \mathrm{~V}_{3}$ (Inorganic BRRI dhan66). The lowest harvest index (45.04\%) was recorded from $\mathrm{F}_{2} \mathrm{~V}_{2}$ (compost 20t ha-1 BRRI dhan56).

Table 7. Effect of fertilization on yield parameters of different varieties of rice

\begin{tabular}{|c|c|c|c|c|c|c|}
\hline \multicolumn{2}{|c|}{ Treatments } & \multicolumn{5}{|c|}{$\begin{array}{l}\text { Yield parameters } \\
\end{array}$} \\
\hline \multicolumn{2}{|c|}{ Varieties } & $\begin{array}{c}\text { Panicle } \\
\text { length }(\mathrm{cm})\end{array}$ & $\begin{array}{c}\text { Grain yield } \\
\mathrm{g} \mathrm{pot}^{-1}\end{array}$ & $\begin{array}{l}1000 \text { grain } \\
\text { weight }(\mathrm{g})\end{array}$ & $\begin{array}{c}\text { Straw } \\
\text { yield } \\
\left(\mathrm{g} \mathrm{pot}^{-1}\right)\end{array}$ & $\begin{array}{c}\text { Harvest } \\
\text { index (\%) }\end{array}$ \\
\hline \multicolumn{2}{|c|}{$\mathrm{V}_{1=\text { Binadhan-7 }}$} & $22.9 a$ & 21.63 & 20.80 & 16.09 & 57.03 \\
\hline \multicolumn{2}{|c|}{$\mathrm{V}_{2=} \mathrm{BRRI}$ dhan 56} & $22.49 a$ & 17.74 & 20.11 & 16.66 & 50.47 \\
\hline \multicolumn{2}{|c|}{$\mathrm{V}_{3=}=\mathrm{BRRI}$ dhan66 } & $20.48 b$ & 19.49 & 22.26 & 15.50 & 54.79 \\
\hline \multicolumn{2}{|c|}{ Level of significance } & $* *$ & NS & NS & NS & NS \\
\hline \multicolumn{7}{|c|}{ Fertilization } \\
\hline \multirow{4}{*}{\multicolumn{2}{|c|}{$\begin{array}{l}\mathrm{F}_{1}=\text { Inorganic } \\
\mathrm{F}_{2}=\text { Compost } 20 \text { tha }^{-1} \\
\mathrm{~F}_{3}=\text { Compost } 30 \text { tha }^{-1} \\
\text { Level of significance }\end{array}$}} & 22.60 & $23.61 a$ & $21.28 \mathrm{ab}$ & $18.19 a$ & $56.19 a$ \\
\hline & & 21.85 & $14.32 \mathrm{~b}$ & $19.66 b$ & $15.3 b$ & $47.89 b$ \\
\hline & & 21.42 & $20.92 a$ & $22.23 a$ & $14.76 b$ & $58.2 \mathrm{a}$ \\
\hline & & NS & ** & $* *$ & $* *$ & $*$ \\
\hline \multicolumn{2}{|c|}{ Level of significance } & & Interact & & & \\
\hline \multirow{4}{*}{$\mathrm{F}_{1}$} & $\mathrm{~V}_{1}$ & $24.16 a$ & $24.28 \mathrm{ab}$ & $20.9 \mathrm{ab}$ & $18.17 \mathrm{a}$ & $57 \mathrm{abc}$ \\
\hline & $\mathrm{V}_{2}$ & $22.5 \mathrm{abc}$ & $16.71 \mathrm{bcd}$ & $19.27 b$ & $16.03 \mathrm{abc}$ & $51.31 \mathrm{abc}$ \\
\hline & $\mathrm{V}_{3}$ & $22.05 \mathrm{abc}$ & $23.91 \mathrm{ab}$ & $22.23 \mathrm{ab}$ & $14.07 \mathrm{bc}$ & $62.78 \mathrm{a}$ \\
\hline & $\mathrm{V}_{1}$ & $22.97 \mathrm{ab}$ & $21.98 \mathrm{ab}$ & $20.15 \mathrm{ab}$ & $18.6 a$ & $53.54 \mathrm{abc}$ \\
\hline \multirow{3}{*}{$\mathrm{F}_{2}$} & $\mathrm{~V}_{2}$ & $22.11 \mathrm{abc}$ & $11.98 \mathrm{~d}$ & $19.3 b$ & $14.67 \mathrm{abc}$ & $45.04 c$ \\
\hline & $\mathrm{V}_{3}$ & $22.38 \mathrm{abc}$ & $19.26 \mathrm{abcd}$ & $20.89 \mathrm{ab}$ & $16.7 \mathrm{abc}$ & $52.82 \mathrm{abc}$ \\
\hline & $\mathrm{V}_{1}$ & $20.66 b c$ & $24.58 \mathrm{a}$ & $22.8 \mathrm{a}$ & $17.8 \mathrm{ab}$ & $58.04 \mathrm{ab}$ \\
\hline \multirow{2}{*}{$\mathrm{F}_{3}$} & $\mathrm{~V}_{2}$ & $20.94 b c$ & $14.29 \mathrm{~cd}$ & $20.42 \mathrm{ab}$ & $15.2 \mathrm{abc}$ & $47.33 b c$ \\
\hline & $\mathrm{V}_{3}$ & $19.83 c$ & $19.59 \mathrm{abc}$ & $23.57 \mathrm{a}$ & $13.5 c$ & $59.01 \mathrm{ab}$ \\
\hline \multicolumn{2}{|c|}{ Level of significance } & *** & ** & *** & $* *$ & $* *$ \\
\hline \multicolumn{2}{|c|}{$\mathrm{CV}(\%)$} & 7.14 & 19.16 & 7.66 & 15.72 & 13.26 \\
\hline
\end{tabular}

In a column, means followed by a different letter(s) differed significantly by Duncan's Multiple Range Test at $P \leq 5 \%$ level of probability.

NS indicates insignificant

*indicates significant at 5\% level of probability

**indicates significant at $1 \%$ level of probability 


\section{Soil chemical properties}

Results revealed that organic matter was increased with the use of different composts treatment (Table 8$)$. The highest organic matter $(2.328 \%)$ and the highest organic carbon $(1.29 \%)$ were found in the case of $\mathrm{F}_{3}$ (compost $30 \mathrm{t} \mathrm{ha}^{-1}$ ). The lowest organic matter (1.03\%) and the lowest organic carbon $(0.49 \%)$ were found in the case of $\mathrm{F}_{1}$ (inorganic). The change of $\mathrm{pH}$ value was insignificant by the application of fertilization. Results revealed that N, P, K, S content of the soil was increased with the use of different composts treatment. The highest $\mathrm{N}(0.102 \%), \mathrm{P}$ (130.0 $\mu \mathrm{g} / \mathrm{g}$ soil), $\mathrm{K}\left(0.449 \mathrm{ppm} / 100 \mathrm{~g}\right.$ soil), $\mathrm{S}\left(62.04 \mu \mathrm{g} / \mathrm{g}\right.$ soil) was found in $\mathrm{F}_{3}$ (compost $30 \mathrm{t}$ $\left.\mathrm{ha}^{-1}\right)$ treatment. The lowest $\mathrm{N}(0.0856 \%), \mathrm{P}(110.0 \mu \mathrm{g} / \mathrm{g}$ soil), $\mathrm{K}(0.37 \mathrm{ppm} / 100 \mathrm{~g}$ soil), $\mathrm{S}$ $\left(39.92 \mu \mathrm{g} / \mathrm{g}\right.$ soil) was found in $\mathrm{F}_{1}$ (inorganic) treatment. A similar trend was also found by Liza et al. (2014) and Salaque et al. (2004) due to the application of organic manure.

Table 8. Effect of fertilization on soil chemical properties

\begin{tabular}{|c|c|c|c|c|c|c|c|}
\hline \multicolumn{8}{|c|}{ Treatments } \\
\hline Fertilization & $\mathrm{pH}$ & $\begin{array}{c}\text { Organic } \\
\text { matter (\%) }\end{array}$ & $\begin{array}{c}\text { Organic } \\
\text { carbon } \\
(\%)\end{array}$ & $\mathrm{N}(\%)$ & $\begin{array}{c}\mathrm{P} \\
(\mu \mathrm{g} / \mathrm{g} \\
\text { soil) }\end{array}$ & $\begin{array}{c}\mathrm{K} \\
\text { (ppm/100 } \\
\mathrm{g} \text { soil) }\end{array}$ & $\begin{array}{c}\mathrm{S} \\
(\mu \mathrm{g} / \mathrm{g} \\
\text { soil) }\end{array}$ \\
\hline $\mathrm{F}_{1}=$ Inorganic & 6.529 & $1.030 \mathrm{c}$ & $0.49 \mathrm{~b}$ & 0.0856 & $110.0 \mathrm{~b}$ & $0.37 b$ & $39.92 c$ \\
\hline $\mathrm{F}_{2}=$ Compost 20 tha $^{-1}$ & 6.650 & $1.912 b$ & $1.13 a$ & 0.090 & $126.4 \mathrm{a}$ & $0.436 a$ & $49.24 b$ \\
\hline $\mathrm{F}_{3}=$ Compost 30 tha $^{-1}$ & 6.618 & $2.328 \mathrm{a}$ & $1.289 a$ & 0.102 & $130.0 \mathrm{a}$ & $0.449 a$ & $62.04 a$ \\
\hline Level of significance & NS & *** & $* *$ & NS & *** & *** & $* *$ \\
\hline CV (\%) & 1.85 & 20.78 & 22.73 & 22.09 & 6.72 & 8.02 & 11.94 \\
\hline
\end{tabular}

In a column, means followed by a different letter(s) differed significantly by Duncan's Multiple Range Test at $P \leq 5 \%$ level of probability.

NS indicates insignificant

*indicates significant at $5 \%$ level of probability

**indicates significant at $1 \%$ level of probability

\section{Conclusion}

According to the present study, it could be suggested that the rice is grown with Compost-30 $t$ $\mathrm{ha}^{-1}$ along with var.Binadhan-7) revealed to get the optimum yield. However, these findings need to be further investigated and evaluated in the different agro-ecological zone before the final recommendation for the farmers.

\section{References}

Alam, M.J. 2017. Amelioration of the adverse effect of drought on rice cultivation through poultry litter based compost.MS thesis, Department of Crop Physiology and Ecology, Hajee Mohammad Danesh Science and Technology University, Dinajpur-5200, Bangladesh.

Alam, M.S., M.A. Baki, M.S. Sultana, K.J. Ali and M.S. Islam.2012.Effect of variety, spacing and number of seedlings per hill on the yield potentials of transplant aman rice. IJARIT. 2 (12): $10-15$.

Aziz, M.A. 2008. Effect of cowdung on the growth and yield of hybrid and HYV rice.MS Thesis, Department of Agronomy, Bangladesh Agricultural University, Mymensingh.

BBS (Bangladesh Bureau of Statistics).2018. Statistical Yearbook of Bangladesh. Statistics Division, Ministry of Planning, Government of the People's Republic of Bangladesh. Dhaka. Bangladesh. 
BBS (Bangladesh Bureau of Statistics). 2015. Statistical Yearbook of Bangladesh. Statistics Division, Ministry of Planning, Government of the People's Republic of Bangladesh. Dhaka, Bangladesh.

Bair, W. 1990. Characterization of the environment for sustainable agriculture in Semi-arid Tropics. In: Proc. Sustainable Agriculture Issue, Prospective and Prospects in Semi-arid Tropics (ed. Singh, R.P.), Hyderabad, India. India Soc. Agron. pp.90-128.

Buri, M.M., R.N. Issaka, T. Wakatsuki and E. Otoo. 2006. Soil organic amendments and mineral fertilizers: options for sustainable lowland rice production in the forest agro-ecology of Ghana. Agril. Food Sci. J. Ghana, 3(1): 237-248.

Chowdhury, A.K.M.M.B., M.A. Hossain, J. Alam, M.A. Hasan and M.Z. Islam. 2020. Amelioration of adverse effect of drought on BRRI dhan28 through poultry litter based compost. The Agriculturists 18(1): 42-55.

Chowdhury, A.K.M.M.B., C.S. Akratos, D.V. Vayenas and S. Pavlou. 2013. Olive mill waste composting: a review. Int. Biodeter Biodegr. 85: 108-119.

Diacono, M. and F. Montemurro. 2010. Long-term effects of organic amendments on soil fertility. A review. Agron. Sustain. Dev. 30: 401-422.

Golabi, M.H., M.J. Denney and C. Iyekar. 2007. Value of composted organic wastes as an alternative to synthetic fertilizers for soil quality improvement and increased yield. Compost Sci. Utilization, 15(4): 267-271.

Gomez, K.A. and A.A. Gomez. 1984. Statistical Procedure for Agricultural Research. International Rice Research Institute. John Wiley and Sons, New York pp.139-240.

Haque, A. 2013. Integrated use of manures and fertilizers for maximizing the growth and yield of Boro rice (cv. BRRI dhan28). MS Thesis, Department of Soil Science, Bangladesh Agricultural University, Mymensingh.

Hossaen, M.A., A.T.M. Shamsuddoha, A.K. Paul, M.S.I. Bhuiyan and A.S.M. Zobaer.2011. Efficacy of different organic manures and inorganic fertilizer on the yield and yield attributes of Boro rice. The Agriculturists 9(1\&2): 117-125.

Hossain, M.I., M.N. Uddin, M.S. Islam, M.K. Hossain and M.A.H. Khan. 2010. Effects of manures and fertilizer on nutrient content and uptake by BRRI dhan 29. J. Agroforest. Environ. 3(2): 65-67.

Islam, F., M.A. Khan, A.S.M.F. Bari, M.T. Hosain and Sabikunnaher, M. 2013. Effect of fertilizer and manure on the growth, yield and grain nutrient concentration of Boro Rice (Oryza sativa L.) under different water management practices. The Agriculturists, 11: 44-51.

Islam, M.R., M.B. Rashid, A.B. Siddique and H. Afroz. 2014. Integrated effects of manures and fertilizers on the yield and nutrient uptake by BRRI dhan49. J. Bangladesh Agril. Univ. 12(1): 67-72.

Kobra, U.E.Z. 2016. Valorization of poultry wastes by aerobic composting for the organic agriculture. MS Thesis, Department of Crop Physiology and Ecology, Hajee Mohammad Danesh Science and Technology University Dinajpur-5200, Bangladesh.

Liza, M.M.J., M.R. Islam, M. Jahiruddin, M.M. Hasan, M.A. Alam, S.M. Shamsuzzaman and A.W. Samsuri. 2014. Residual effects of organic manures with different levels of chemical fertilizers on rice. Life Sci. J. 11(12): 6-12.

Meelu, O.P. and Y. Singh. 1991. Integrated use of fertilizers and organic manure for higher returns. Prog. Farm. Punjab Agril. Univ. 27: 3-4.

Najafi, N. and M.A. Abbasi. 2013. Effects of soil water conditions, sewage sludge, poultry manure and chemical fertilizers on macronutrients concentrations in rice plant. Int. J. Agron. Plant Prodn. 4(5): 1066-1077. 
Nambiar, K.K.M. 1991. Long-term fertility effects on wheat productivity. In: wheat for the Nontraditional Warm areas, (ed.) Saunders D.A., CIMMYT. pp.516-521.

Rejesus, R.M., M.E.M. Mutuc, M. Yasar, A.V. Lapitan, F.G. Palis and T.T.N. Chi. 2012. Sending Vietnamese rice farmers back to school: further evidence on the impacts of farmer field schools. Canadian J. Agric. Econ. 60(3): 407-426.

Saha, P.K. and M.A.M. Miah. 2009. Efficiency of IPNS-based chemical fertilizer application in wet land rice. Bangladesh J. Agric. Res. 34(1): 5-13.

Saha, U. 2014. Integrated use of cowdung and inorganic fertilizer on the performance of modern varieties of transplanted aman rice. MS Thesis, Department of Agronomy, Bangladesh Agricultural University, Mymensingh, Bangladesh.

Saleque, M.A., M.S. Abedin, N.I. Bhuiyan, S.K. Zaman and G.M. Panaullah. 2004. Longterm effect of inorganic and organic fertilizer sources on yield and nutrient accumulation of lowland rice. Field Crops Res. 86: 53-65.

Sarker, B.C., M. Zahan, U.K. Majumder, M.A. Islam and B. Roy. 2013. Growth and yield potential of some local and high yielding Boro rice cultivars. J. Agroforest. Environ. 7(1): 107-110.

Shelley, I.J., M. Takahashi-Nosaka, M. Kano-Nakata, M.S. Haque and Y. Inukai. 2016. Rice cultivation in Bangladesh: Present scenario, problems and prospects. J. Intl. Coop. Agric. Dev. 14: 20-29.

Tilahun, T., D. Nigussie, B. Wondimu and G. Setegn. 2013. Effect of farmyard manure and inorganic fertilizers on the growth, yield and moisture stress tolerance of rain-fed lowland rice. American J. Res. Comm. 1(4): 275-301.

Urra, J., I. Alkorta and C. Garbisu. 2019. Potential benefits and risks for soil health derived from the use of organic amendments in agriculture. Agron. 9(9): 542-552.

Zubaer, M.A., A.K.M.M.B. Chowdhury, M.Z. Islam, T. Ahmed and M.A. Hasan. 2007. Effects of water stress on growth and yield attributes of aman rice genotypes. Intl. J. Sust. Crop Prodn. 2(6): 25-30. 